Honam Mathematical J. 34 (2012), No. 2, pp. 219-230

http://dx.doi.org/10.5831/HMJ.2012.34.2.219

\title{
THE CORRELATION DIMENSION OF GENERALIZED CANTOR-LIKE SETS
}

\author{
Mi Ryeong Lee And Hunki Baek
}

\begin{abstract}
In the paper, a symbolic construction is considered to define generalized Cantor-like sets. Lower and upper bounds for the correlation dimension of the sets with a regular condition are obtained with respect to a probability Borel measure. Especially, for some special cases of the sets, the exact formulas of the correlation dimension are established and we show that the correlation dimension and the Hausdorff dimension of some of them are the same. Finally, we find a condition which guarantees the positive correlation dimension of the generalized Cantor-like sets.
\end{abstract}

\section{Introduction}

The most basic tool to characterize fractal sets is to calculate their fractal dimensions, such as the Hausdorff dimension, information dimension, and box dimension, etc. Especially, the Hausdorff dimension has been investigated by many researchers because it has the advantage of being defined for any set, and is mathematically convenient, as it a based on measures, which are relatively easy to manipulate.(cf, $[7,15,16])$. However, one of the disadvantage of studying the Hausdorff dimension is that in many cases it is very difficult to compute or to estimate its value.(see [6]). In order to overcome such drawback, in [8], the authors introduced a brand new fractal dimension, so called the correlation dimension. The correlation dimension rather than other dimensions often give us as well advantages of calculations as mathematical characterizations and some physical explanations on a dynamical system as the information or box dimension.(see $[8,9,11])$.

Received March 14, 2012. Accepted March 29, 2012.

2000 Mathematics Subject Classification. 28A80, 37B10.

Key words and phrases. correlation dimension, generalized Cantor-like set.. 
Many authors have studied for various types of Cantor sets as typical fractal sets which mean compact, totally disconnected and perfect subsets in $\mathbb{R}^{1}$. (cf, $\left.[1,2,4,5,13,14,19]\right)$. The author in [1] found out lower and upper bounds for the Hausdorff dimension of a deranged Cantor set and the authors in [2] and [19] calculated the exact packing dimension and Hausdorff dimension, respectively. In [4], they found the Hausdorff dimension of a Cantor set considering infinitely many contractive ratios. On the other hand, in [5] and [13] they considered a random map having a given number of contractive ratios, in connection with each random sequence in $(0,1)$ and obtained formulas for the correlation dimension of the generated set by the given map. Especially, in [14] and [18], the authors introduced a symbolic construction for Cantor-like sets which are generalizations of constructions, so-called, the iterated function system of a family of contractive maps or the Moran construction and so on. In addition, they investigated formulas for fractal dimensions, such as Hausdorff, box, packing or correlation dimensions, of the generated set by a symbolic construction. In order to obtain lower and upper bounds for the dimensions of the Cantor-like set, they used an equilibrium measure on the set concerned with a suitable vector of real numbers.

Thus, in this paper, we first introduce a symbolic construction for generalized Cantor-like sets. We deal with infinitely many contractive ratios in the construction of the sets and define a probability Borel measure on the set induced by the given symbolic construction together with a sequence of vectors of random numbers in $(0,1)$. Using the theory of energy, we find lower and upper bounds for the correlation dimension of the generalized Cantor-like sets with a regular condition with respect to the probability measure. Moreover, we obtain results in [4] as corollaries by considering some restrictions in our construction and show that the correlation dimension of some of the sets is equal to the Hausdorff dimension. Also, we see that many results about the correlation dimension in other papers can be followed as corollaries from our results. Finally, we find a condition which guarantees the positive correlation and Hausdorff dimensions of some generalized Cantor-like sets.

\section{Preliminaries}

Let us introduce a symbolic construction for generalized Cantor-like sets in $\mathbb{R}^{1}$. Fix an integer $l \geq 2$ and take an integer $m$ satisfying $1 \leq$ $m \leq l$. 
Let $\Sigma_{l} \equiv\{1,2, \ldots, l\}^{\mathbb{N}}=\left\{\left(i_{1}, i_{2}, \ldots\right): i_{j} \in\{1, \ldots, l\}, j=1,2, \ldots\right\}$. Consider a symbolic set $\mathcal{S}=\left\{\left(i_{1}, i_{2}, \ldots\right) \in \Sigma_{l}: i_{2 n-1} \in\{1, \ldots, m\}, i_{2 n} \in\right.$ $\{1, \ldots, l\}, n=1,2, \ldots\}$.

Put $I=[0,1]$. Denote $|\cdot|$ the length of an interval in $\mathbb{R}^{1}$.

Let us construct the basic intervals of a generalized Cantor-like set as follows. $I$ contains mutually disjoint closed subintervals $I_{1}, \ldots, I_{m}$ satisfying $0<\left|I_{i}\right|=r_{i}$ and $\sum_{i=1}^{m} r_{i}<1$. Next, $I_{i}$ contains mutually disjoint closed subintervals $I_{i 1}, I_{i 2}, \ldots, I_{i l}$ satisfying

$$
0<\frac{\left|I_{i j}\right|}{\left|I_{i}\right|}=r_{i j} \text { and } \sum_{j=1}^{l} r_{i j}<1
$$

for $1 \leq i \leq m$ and $1 \leq j \leq l$. By repeating these subdivisions, each basic $I_{i_{1} \ldots i_{n-1}}$ for $n \geq 2$ contains mutually disjoint closed subintervals $\left\{I_{i_{1} \ldots i_{n-1} i_{n}}\right\}$ satisfying

$$
0<\frac{\left|I_{i_{1} \ldots i_{n}}\right|}{\left|I_{i_{1} \ldots i_{n-1}}\right|}=r_{i_{1} \ldots i_{n}} \text { and } \sum_{i_{n}} r_{i_{1} \ldots i_{n}}<1
$$

where $1 \leq i_{n} \leq m$ for $n$ being odd and $1 \leq i_{n} \leq l$ for $n$ being even number.

For each $n \in \mathbb{N}$, an $n$-tuple $\left(i_{1}, i_{2}, \ldots, i_{n}\right) \in\{1,2, \ldots, l\}^{n}$ is called $\mathcal{S}$ admissible if there exists $w=\left(i_{1}^{\prime}, i_{2}^{\prime}, \ldots\right) \in \mathcal{S}$ such that $i_{j}^{\prime}=i_{j}$ for $1 \leq$ $j \leq n$. Put $\mathcal{S}_{n}$ be the set of all $\mathcal{S}$-admissible $n$-tuples $\left(i_{1}, \ldots, i_{n}\right)$ and $\mathcal{S}^{*}=\cup_{n \geq 1} \mathcal{S}_{n}$.

We may assume that the following condition;

$$
0<\underline{r} \leq \inf \left\{r_{i_{1} \ldots i_{n}}:\left(i_{1}, \ldots, i_{n}\right) \in \mathcal{S}^{*}, \text { for } n \in \mathbb{N}\right\} .
$$

Let

$$
F=\bigcap_{n=1}^{\infty} \bigcup_{\left(i_{1}, \ldots, i_{n}\right) \in \mathcal{S}_{n}} I_{i_{1} i_{2} \ldots i_{n}}
$$

We call this set $F$ a generalized Cantor-like set generated by the symbolic set $\mathcal{S}$.

REMARK 2.1 In this note, because we do not use properties of ergodicity as in [13] or [14], we need not conditions about the symbolic set, so-called, invariant set and topological transitivity under some shift map. $F$.

Now we are in a position to define a probability measure on the set 
First, we choose $m$-numbers $c_{1}^{(1)}, c_{2}^{(1)}, \ldots, c_{m}^{(1)} \in(0,1)$ satisfying $\Sigma_{k=1}^{m} c_{k}^{(1)}$ $=1$, and define a map $p$ by $p\left(I_{i}\right)=c_{i}^{(1)}$ for $i=1, \ldots, m$. Next, we choose $l$-numbers $c_{1}^{(2)}, c_{2}^{(2)}, \ldots, c_{l}^{(1)} \in(0,1)$ satisfying $\Sigma_{k=1}^{l} c_{k}^{(2)}=1$, and define $p\left(I_{i_{1} i_{2}}\right)=c_{i_{2}}^{(2)} \cdot p\left(I_{i_{1}}\right)=c_{i_{2}}^{(2)} c_{i_{1}}^{(1)}$ for $i_{1}=1, \ldots, m$ and $i_{2}=1,2, \ldots, l$. Continuing these processes, at $n$-th stage, we can choose either $m$-numbers $c_{1}^{(n)}, c_{2}^{(n)}, \ldots, c_{m}^{(n)} \in(0,1)$ satisfying $\Sigma_{k=1}^{m} c_{k}^{(n)}=1$ for $n$ being odd number or $l$-numbers $c_{1}^{(n)}, c_{2}^{(n)}, \ldots, c_{l}^{(n)} \in(0,1)$ satisfying $\Sigma_{k=1}^{l} c_{k}^{(n)}=1$ for $n$ being even number. And we define a map $p$ as follows:

$$
p\left(I_{i_{1} \ldots i_{n}}\right)=c_{i_{n}}^{(n)} \cdot p\left(I_{i_{1} \ldots i_{n-1}}\right)=\prod_{j=1}^{n} c_{i_{j}}^{(j)}
$$

for each $\mathcal{S}$-admissible $n$-tuples $\left(i_{1}, i_{2}, \ldots, i_{n}\right)$ and all $n \in \mathbb{N}$.

For the generalized Cantor-like set $F$ generated by $\mathcal{S}$, put

$$
\mu_{p}(A)=\inf \left\{\sum_{\left(i_{1}, \ldots, i_{n}\right) \in \mathcal{C}} p\left(I_{i_{1} \ldots i_{n}}\right): A \subset \bigcup_{\left(i_{1}, \ldots, i_{n}\right) \in \mathcal{C}} I_{i_{1} \ldots i_{n}} \text { for } \mathcal{C} \subset \mathcal{S}^{*}\right\},
$$

for any Borel subset $A$ of $F$.

Then $\mu_{p}$ is a probability measure on the set $F$ (see $[3,4]$ ).

To obtain the lower bound of the correlation dimensions of the generated Cantor-like set generated by the symbolic set $\mathcal{S}$, we a little moderate in our construction by adaptation a regular symbolic construction in [14] or [18].

Let $Z \subset \mathcal{S}$. Given $0<r<1$. For each $\mathbf{i}=\left(i_{1}, i_{2}, \ldots, i_{n}, \ldots\right) \in Z$, we can find the unique integer number $n(\mathbf{i})$ such that $r_{i_{1}} \cdots r_{i_{n(\mathbf{i})+1}} \leq r<$ $r_{i_{1}} \cdots r_{i_{n(\mathbf{i})}}$. We note that $n(\mathbf{i}) \rightarrow \infty$ as $r \rightarrow 0$. Fix $\mathbf{i} \in Z$ and consider the cylinder set $C_{i_{1}, i_{2}, \ldots, i_{n(\mathbf{i})}}$,

$$
C_{i_{1}, i_{2}, \ldots, i_{n(\mathbf{i})}}=\left\{\mathbf{j}=\left(j_{1}, j_{2}, \ldots\right) \in \mathcal{S}: j_{k}=i_{k}, k=1,2, \ldots, n(\mathbf{i})\right\} .
$$

Plainly, $\mathbf{i} \in C_{i_{1}, i_{2}, \ldots, i_{n(\mathbf{i})}}$. We note that if $\mathbf{i}^{\prime} \in C_{i_{1}, i_{2}, \ldots, i_{n\left(\mathbf{i}^{\prime}\right)}} \cap Z$ and $n\left(\mathbf{i}^{\prime}\right) \geq n(\mathbf{i})$, then $C_{i_{1}, i_{2}, \ldots, i_{n\left(\mathbf{i}^{\prime}\right)}} \cap Z \subset C_{i_{1}, i_{2}, \ldots, i_{n(\mathbf{i})}} \cap Z$.

Let $C(\mathbf{i})$ be the largest cylinder set containing $\mathbf{i}$ with the property that $C(\mathbf{i})=C_{i_{1}, \cdots, i_{n\left(\mathbf{i}^{\prime \prime}\right)}}$ for some $\mathbf{i}^{\prime \prime} \in C(\mathbf{i})$ and $C_{i_{1}, \cdots, i_{n\left(\mathbf{i}^{\prime}\right)}} \cap Z \subset C(\mathbf{i}) \cap Z$ for any $\mathbf{i}^{\prime} \in C(\mathbf{i}) \cap Z$. Then the sets $C(\mathbf{i})$ corresponding to different $\mathbf{i} \in Z$ either coincide or are disjoint. We denote these sets by $C_{r}^{(j)}$, $j=1,2, \cdots, N_{r}$. We can easily see that these sets form a cover of $Z$. 
Now, we define a bijective map $\Pi$ from the symbolic set $\mathcal{S}$ to $F$ as $\Pi(\mathbf{i})=\cap_{n=1}^{\infty} I_{i_{1} i_{2} \cdots i_{n}} \in F$ for any $\mathbf{i}=\left(i_{1}, i_{2}, \cdots\right) \in \mathcal{S}$.

Put $I_{r}^{(j)}=\Pi\left(C_{r}^{(j)}\right)$. Let $N(x, r)$ denote the number of basic intervals $I_{r}^{(j)}$ that have non-empty intersection with the open ball $B(x, r)$ centered $x$ and radius $r$. If $N(x, r)$ is less than a uniform constant in $x$ and $r$, then we call this a regular condition. And we call a symbolic construction regular if it admits the regular condition(cf. [14, 18]).

REMARK 2.2 For the general case $l \supsetneqq m$, if the basic intervals $\left\{I_{i_{1} i_{2} \ldots i_{n}}\right\}$ satisfy the following condition

$$
\sup _{n} \sup _{\left(i_{1}, i_{2}, \ldots, i_{n}\right) \in \mathcal{S}_{n}}-\frac{\log \left|I_{i_{1} i_{2} \ldots i_{n}}\right|}{n} \leq C
$$

for some constant $C>0$, then the symbolic construction becomes regular.

\section{Results}

Throughout this paper, let $\mathcal{S}, F, \Pi$ and $\mu_{p}$ be the same as in section 2. For simplicity of notations, we write $\mathbf{i}_{n}$ for the $n$-tuple $\left(i_{1}, \ldots, i_{n}\right) \in$ $\{1,2, \ldots, l\}^{n}$ for $\mathbf{i}=\left(i_{1}, \ldots, i_{n}, \ldots\right) \in \Sigma_{l}$ and $I_{\mathbf{i}_{n}}$ for the basic interval $I_{i_{1}, i_{2}, \ldots, i_{n}}$ at the $n$-th $\operatorname{step}(n \geq 1)$. Also we write $\mu$ for $\mu_{p}$.

Our goal is to obtain some estimations about the correlation dimension $D_{2}(A, \mu)$ of $A(\subset \mathbb{R})$ with respect to the probability measure $\mu([5,17$, $18])$;

$$
D_{2}(A, \mu) \equiv \sup \left\{s \geq 0: I_{s}(\mu)<\infty\right\}=\inf \left\{s \geq 0: I_{s}(\mu)=\infty\right\},
$$

where $I_{s}(\mu)=\int_{A} \int_{A}|x-y|^{-s} d \mu(x) d \mu(y)$ is the $s$-energy of $A$ with respect to $\mu$.

Denote $B(x, r)$ for the open ball of radius $r>0$ centered at $x$.

Lemma 3.1. Let $F$ be a generalized Cantor-like set. If $E \subseteq F$ satisfies that for $\mu$-almost all $\cap_{n=1}^{\infty} I_{\mathbf{i}_{n}}(\equiv x) \in E$,

$$
\limsup _{n \rightarrow \infty} \frac{\log \mu\left(I_{\mathbf{i}_{n}}\right)}{\log \left|I_{\mathbf{i}_{n}}\right|} \leq \beta
$$

then there exists a constant $a_{1}>0$ such that $\mu(B(x, r) \cap E) \geq a_{1} r^{\beta}$.

Proof. Let $r>0$ be given. For each $x \in E$, there uniquely exists $\mathbf{i}=\left(i_{1}, i_{2}, i_{3}, \ldots\right) \in \mathcal{S}$ satisfying $\Pi(\mathbf{i})=\cap_{n=1}^{\infty} I_{\mathbf{i}_{n}}=x$. 
Using the similar arguments for the cylinder set as previous, we can find a large number $n_{0} \equiv n_{0}(x) \in \mathbb{N}$ satisfying $x \in I_{\mathbf{i}_{n_{0}+1}}$ and

$$
\left|I_{\mathbf{i}_{n_{0}+1}}\right| \leq r<\left|I_{\mathbf{i}_{0}}\right| .
$$

By the assumption, there exists a large number $N_{0}$ such that for any $n \geq N_{0}$,

$$
x \in I_{\mathbf{i}_{n}} \text { and } \mu\left(I_{\mathbf{i}_{n}}\right) \geq\left|I_{\mathbf{i}_{n}}\right|^{\beta} .
$$

For a sufficiently small number $0<r<1$, we may assume that $n_{0} \geq N_{0}$. Then for $\mu$-almost all $x \in E$, we have $B(x, r) \supset I_{\mathbf{i}_{n_{0}+1}}$ and using (1) and (2),

$$
\begin{aligned}
\mu(B(x, r) \cap E) & \geq \mu\left(I_{\mathbf{i}_{n_{0}+1}}\right) \geq\left|I_{\mathbf{i}_{n_{0}+1}}\right|^{\beta} \\
& \geq \frac{\left|I_{\mathbf{i}_{n_{0}+1}}\right|^{\beta}}{\left|I_{\mathbf{i}_{0}}\right|^{\beta}} \cdot r^{\beta} \geq \underline{r}^{\beta} \cdot r^{\beta} \equiv a_{1} \cdot r^{\beta} .
\end{aligned}
$$

Theorem 3.2. Let $F$ be a generalized Cantor-like set. If $E \subseteq F$ satisfies the same condition as in Lemma 3.1, then we have $D_{2}(E, \mu) \leq \beta$.

Proof. In order to obtain the upper bound $\beta$ for the correlation dimension $D_{2}(E, \mu)$, we need to calculate the energy of $E$ with respect to the measure $\mu$. Put $\phi_{t}(x)=\int_{E}|x-y|^{-t} d \mu(y)$. Then, by Lemma 3.1, for all $t>\beta$,

$$
\begin{aligned}
\phi_{t}(x) & =\int_{0}^{\infty} \mu\left(\left\{y \in E:|x-y|^{-t} \geq r\right\}\right) d r=\int_{0}^{\infty} \mu\left(B\left(x, r^{-1 / t}\right) \cap E\right) d r \\
& =t \int_{0}^{\infty} \epsilon^{-t-1} \mu(B(x, \epsilon) \cap E) d \epsilon \\
& \geq t \int_{0}^{\infty} \epsilon^{-t-1} \cdot a_{1} \cdot \epsilon^{\beta} d \epsilon=\infty .
\end{aligned}
$$

Therefore $I_{t}(\mu)=\int_{E} \phi_{t}(x) d \mu(x)=\infty$, for all $t>\beta$, which implies $D_{2}(E, \mu) \leq \beta$.

Lemma 3.3. Let $F$ be a generalized Cantor-like set generated by a regular symbolic construction. If $E \subseteq F$ satisfies that for $\mu$-almost all $x\left(\equiv \cap_{n=1}^{\infty} I_{\mathbf{i}_{n}}\right) \in E$,

$$
\alpha \leq \liminf _{n \rightarrow \infty} \frac{\log \mu\left(I_{\mathbf{i}_{n}}\right)}{\log \left|I_{\mathbf{i}_{n}}\right|}
$$

then there exists a constant $a_{2}>0$ such that $\mu(B(x, r) \cap E) \leq a_{2} r^{\alpha}$. 
Proof. Let $r>0$ be given. For each $x \in E$, there uniquely exists $\mathbf{i}=\left(i_{1}, i_{2}, i_{3}, \ldots\right) \in \mathcal{S}$ satisfying $\Pi(\mathbf{i})=\cap_{n=1}^{\infty} I_{\mathbf{i}_{n}}=x$. Also we can find a number $n_{1} \equiv n_{1}(x) \in \mathbb{N}$ satisfying $x \in I_{\mathbf{i}_{n_{1}+1}}$ and

$$
\left|I_{\mathbf{i}_{n_{1}+1}}\right| \leq r<\left|I_{\mathbf{i}_{n_{1}}}\right| .
$$

From the above assumption, we can get for all $l \geq k$,

$$
x \in I_{\mathbf{i}_{l}} \text { and } \mu\left(I_{\mathbf{i}_{l}}\right) \leq\left|I_{\mathbf{i}_{l}}\right|^{\alpha} .
$$

From the definition of the regular symbolic construction, we note that $(B(x, r) \cap E) \subset \bigcup^{N(x, r)} I_{\mathbf{i}_{n_{1}}}$. If we take $r$ sufficiently small, then we may assume that $n_{1} \geq k$. Hence for $\mu$-almost all $x \in E$, using the facts (3) and (4),

$$
\begin{aligned}
\mu(B(x, r) \cap E) & \leq \sum^{N(x, r)} \mu\left(I_{\mathbf{i}_{n_{1}}}\right) \leq \text { Const. } \cdot\left|I_{\mathbf{i}_{n_{1}}}\right|^{\alpha} \\
& \leq \text { Const. } \underline{r}^{-\alpha} r^{\alpha} \equiv a_{2} r^{\alpha} .
\end{aligned}
$$

Theorem 3.4. Let $F$ be a generalized Cantor-like set generated by a regular symbolic construction. If $E \subseteq F$ satisfies the same condition as in Lemma 3.3, then we have $\alpha \leq D_{2}(E, \mu)$.

Proof. In order to obtain the lower bound $\alpha$ for the correlation dimension $D_{2}(E, \mu)$ of $E$, we need to calculate the $s$-energy of $E$ as in the proof of Theorem 3.2. Using Lemma 3.3, for all $t<\alpha$,

$$
\begin{aligned}
\phi_{t}(x) & =t \int_{0}^{\infty} \epsilon^{-t-1} \mu(B(x, \epsilon) \cap E) d \epsilon \\
& <t\left[\int_{0}^{1} \epsilon^{-t-1} \mu(B(x, \epsilon) \cap E) d \epsilon+\int_{1}^{\infty} \epsilon^{-t-1} \mu(E) d \epsilon\right] \\
& \leq a_{2} t \int_{0}^{1} \epsilon^{\alpha-t-1} d \epsilon+\mu(E)<\infty .
\end{aligned}
$$

Hence $I_{t}(\mu)=\int_{E} \phi_{t}(x) d \mu(x)<\infty$ for all $t<\alpha$, which implies $D_{2}(E, \mu) \geq$ $\alpha$.

REMARK 3.1. In our construction, if let $l=m$ and the ratios at $n$-th step $r_{i_{1}, i_{2}, \ldots, i_{n}} \in\left\{r_{1}, \ldots, r_{m}\right\}$ for $n \geq 1$ as in [5], then such construction in [5] becomes regular and the results in [3] follow.

As the special case $l=m(\geq 2)$, i.e. $\mathcal{S}=\Sigma_{m} \equiv\{1,2, \ldots, m\}^{\mathbb{N}}$, we obtain some interesting results for the central Cantor sets(see [4]) and generalized Cantor-like sets. 
First case, if we take $r_{n+1}=r_{\mathbf{i}_{n}, 1}=\cdots=r_{\mathbf{i}_{n}, m}$ for all $\mathbf{i}_{n}=\left(i_{1}, i_{2}, \ldots, i_{n}\right)$ $\in\{1, \ldots, m\}^{n}, n \geq 1$, then we call the set $F$ the central Cantor set $([4])$. Moreover, if we restrict that the chosen numbers $c_{k}^{(j)}=\frac{1}{m}$, for $1 \leq k \leq m$ and all $j \geq 1$, then we also have the induced probability measure $\bar{\mu}$ on $F$ from the method in section 2 .

Proposition 3.5. Let $F$ be the central Cantor set and $\bar{\mu}$ be defined as the above. Then

$$
D_{2}(F, \bar{\mu})=\liminf _{n \rightarrow \infty} \frac{n \log m}{-\log r_{1} \cdot r_{2} \cdots r_{n}}=\operatorname{dim}_{H} F
$$

where $\operatorname{dim}_{H} F$ is the Hausdorff dimension of the set $F$.

Proof. The proof of the second equality is parallel to the proof of Proposition 3.1 in [4].

Since we know that $D_{2}(F, \bar{\mu}) \leq \operatorname{dim}_{H} F$ for any probability measure $\bar{\mu}$ on $F([13,14,20,21])$, we only prove that

$$
D_{2}(F, \bar{\mu}) \geq \liminf _{n \rightarrow \infty} \frac{n \log m}{-\log r_{1} \cdot r_{2} \cdots r_{n}} \equiv t .
$$

Given $\epsilon>0$, we can find $n_{0} \in \mathbb{N}$ such that $m^{-k}<\left(r_{1} \cdot r_{2} \cdots r_{k}\right)^{t-\epsilon}$ for all $k \geq n_{0}$.

For any $x \in F$, we choose an open ball $B(x, r)$ satisfying $r_{1} \cdot r_{2} \cdots r_{n+1} \leq$ $r<r_{1} \cdot r_{2} \cdots r_{n}$ for such an $n \geq n_{0}$. Put $I_{n}=I_{\mathbf{i}_{n}}\left(\mathbf{i}_{n} \in \mathcal{S}_{n}\right)$ for the basic intervals at the $n$-th step in the construction of $F$. Then the number $N(x, r)$ is less than or equal to 2 . Using the above facts and each $r_{i}<\frac{1}{m}$ for $i=1, \ldots, m$,

$$
\begin{aligned}
\bar{\mu}(B(x, r))=\mu(B(x, r) \cap F) & \leq 2 \cdot \bar{\mu}\left(I_{n}\right)=2 \cdot m^{-n} \\
& <2 \cdot m^{t-\epsilon} \cdot\left(r_{1} \cdot r_{2} \cdots r_{n+1}\right)^{t-\epsilon}<a_{3} r^{t-\epsilon}
\end{aligned}
$$

where $a_{3}>0$ is a constant.

Since $\epsilon$ is arbitrary, we have $\bar{\mu}(B(x, r)) \leq a_{3} r^{t}$, for all $x \in F$ and $r>0$. From Theorem 3.4, we have $D_{2}(F, \bar{\mu}) \geq t$.

Corollary 3.6. If $F$ is the central Cantor set and $\bar{\mu}$ is defined as the above, then the following conditions are equivalent:

(i) $\operatorname{dim}_{H} F=0$;

(ii) $\underline{\lim }_{n \rightarrow \infty}\left(r_{1} \cdot r_{2} \cdots r_{n}\right)^{\frac{1}{n}}=0$;

(iii) $D_{2}(F, \bar{\mu})=0$.

Proof. For (i) $\Longleftrightarrow$ (ii), the argument is similar to Corollary 3.3 in [4]. 
For $\left(\right.$ ii) $\Longleftrightarrow$ (iii), by using two facts $D_{2}(F, \bar{\mu}) \leq \operatorname{dim}_{H} F$ and Proposition 3.5 , we have the equivalence.

Next, for more generalized sets than central Cantor sets, their correlation dimensions are obtained as follows.

Let $F$ be a generalized Cantor-like set generated by a regular symbolic construction and let $p_{k}=c_{j}^{(k)}$ be fixed positive number for $1 \leq k \leq m$ and all $j \geq 1$ satisfying $\sum_{k=1}^{m} p_{k}=1$. We consider the Borel subset $F\left(p_{1}, p_{2}, \ldots, p_{m}\right)$ of $F$ like as in [5] or [13]:

$F\left(p_{1}, \ldots, p_{m}\right) \equiv\left\{\Pi(\mathbf{i}) \in F: \lim _{n \rightarrow \infty} \frac{\#\left\{j: i_{j}=k, 1 \leq j \leq n\right\}}{n}=p_{k}, 1 \leq k \leq m\right\}$.

Denote $\bar{\mu}$ for the induced probability measure on $F\left(p_{1}, \ldots, p_{m}\right)$ from $\left(p_{1}, \ldots, p_{m}\right), \mathbf{i}_{n}=\left(i_{1}, i_{2}, \ldots, i_{n}\right) \in\{1, \ldots, m\}^{n}$ for all $n \geq 1$ and $r_{\mathbf{i}_{n}}$ for the ratios at the $n$-th step (i.e. $\left.r_{\mathbf{i}_{n}}=r_{i_{1} i_{2} \ldots i_{n}}\right)$.

Proposition 3.7. For the subset $F\left(p_{1}, p_{2}, \ldots, p_{m}\right)$ and the probability measure $\bar{\mu}$,

$$
D_{2}\left(F\left(p_{1}, p_{2}, \ldots, p_{m}\right), \bar{\mu}\right)=\liminf _{n \rightarrow \infty} \frac{\sum_{i=1}^{m} p_{i} \log p_{i}}{\frac{1}{n} \log r_{\mathbf{i}_{1}} r_{\mathbf{i}_{2}} \cdots r_{\mathbf{i}_{n}}} .
$$

Proof. By the definition of $F\left(p_{1}, p_{2}, \ldots, p_{m}\right)$, we have for any point $\Pi(\mathbf{i})=\cap_{n=1}^{\infty} I_{\mathbf{i}_{n}}$ in $F\left(p_{1}, \ldots, p_{m}\right)$,

$$
\left|I_{\mathbf{i}_{n}}\right|=r_{\mathbf{i}_{1}} \cdot r_{\mathbf{i}_{2}} \cdots r_{\mathbf{i}_{n}} \quad \text { and } \quad \bar{\mu}\left(I_{\mathbf{i}_{n}}\right)=p_{1}^{n_{1}} \cdot p_{2}^{n_{2}} \cdots p_{m}^{n_{m}}
$$

where $\sum_{i=1}^{m} n_{i}=n$.

Then, by simple calculations as in [5] or [13] and using Theorem 3.2 and 3.4 , we have the result.

Corollary 3.8. In Proposition 3.7, if we take $p_{i}=\frac{1}{m}$ for $i=1, \ldots, m$ and $\bar{\mu}$ as the above, then we obtain the followings

$$
D_{2}\left(F\left(\frac{1}{m}, \ldots, \frac{1}{m}\right), \bar{\mu}\right)=\liminf _{n \rightarrow \infty} \frac{n \log m}{-\log r_{\mathbf{i}_{1}} r_{\mathbf{i}_{2}} \cdots r_{\mathbf{i}_{n}}}=\operatorname{dim}_{H} F\left(\frac{1}{m}, \ldots, \frac{1}{m}\right) .
$$

Now, we introduce the following condition for ratios at each stage which provides a latent possibility for the correlation dimension of a generalized Cantor-like set without regularity. We can obtain the idea and method of proof from [4] and [10].

Proposition 3.9. For every $n$-tuple $\mathbf{i}_{n}=\left(i_{1}, i_{2}, \ldots, i_{n}\right) \in \mathcal{S}^{*}$, if there is a constant $M \in \mathbb{N}$ satisfying for all $n, j \geq 1$,

$$
\frac{1}{M} \leq \frac{r_{\mathbf{i}_{1}} r_{\mathbf{i}_{2}} \cdots r_{\mathbf{i}_{n}}}{r_{\mathbf{i}_{1+j}} r_{\mathbf{i}_{2+j}} \cdots r_{\mathbf{i}_{n+j}}} \leq M
$$


then $\left(r_{\mathbf{i}_{1}} r_{\mathbf{i}_{2}} \cdots r_{\mathbf{i}_{n}}\right)^{\frac{1}{n}}$ converges to a non-zero constant. Then

Proof. Let $\mathbf{i}_{k}=\left(i_{1}, i_{2}, \ldots, i_{k}\right) \in \mathcal{S}^{*}$ for all $k \geq 1$. Put $H_{\mathbf{i}_{k}}=r_{\mathbf{i}_{1}} r_{\mathbf{i}_{2}} \cdots r_{\mathbf{i}_{k}}$.

$$
\frac{1}{M} \leq \frac{H_{\mathbf{i}_{k}} \cdot H_{\mathbf{i}_{j}}}{H_{\mathbf{i}_{k+j}}} \leq M
$$

Taking the logarithm and put $\delta=\log M$ and $L_{\mathbf{i}_{k}}=\log H_{\mathbf{i}_{k}}$ for all $k \geq 1$.

Then

$$
-\delta+L_{\mathbf{i}_{k}}+L_{\mathbf{i}_{j}} \leq L_{\mathbf{i}_{k+j}} \leq \delta+L_{\mathbf{i}_{k}}+L_{\mathbf{i}_{j}} .
$$

It is sufficient to show that $\left\{\frac{L_{\mathbf{i}_{k}}}{k}\right\}$ is a Cauchy sequence.

First, using inequality (5) and inductive method on $k$, we get

$$
-k \delta+k L_{\mathbf{i}_{j}} \leq L_{\mathbf{i}_{k j}} \leq k \delta+k L_{\mathbf{i}_{j}} .
$$

Manipulating (6) inequality, we have

$$
-(k+j) \delta+k L_{\mathbf{i}_{j}} \leq j L_{\mathbf{i}_{k}} \leq(k+j) \delta+k L_{\mathbf{i}_{j}} .
$$

Divide (7) by $k j$, we obtain for all $k$ and $j$,

$$
\left|\frac{L_{\mathbf{i}_{j}}}{j}-\frac{L_{\mathbf{i}_{k}}}{k}\right| \leq\left(\frac{1}{k}+\frac{1}{j}\right) \delta .
$$

Therefore we obtain that $\left\{\frac{L_{\mathbf{i}_{k}}}{k}\right\}$ is a Cauchy sequence.

REMARK 3.2. (1) Let the sequence $\left\{c_{k}^{(j)}\right\}_{j=1}^{\infty}$ be given in section 2 (i.e. $0<c_{k}^{(j)}<1$ for each $j \geq 1$ satisfying $\sum_{k=1}^{m} c_{k}^{(j)}=1(j$ : odd) or $\sum_{k=1}^{l} c_{k}^{(j)}=1(j$ : even $\left.)\right)$. Like as assumptions for the sequence $\left\{c_{k}^{(j)}\right\}_{j=1}^{\infty}$ in Proposition 3.5 or Proposition 3.7, if we have some controls over the sequence $\left\{c_{k}^{(j)}\right\}_{j=1}^{\infty}$, then from Proposition 3.9, we can obtain that two bounds of the correlation dimension for a generalized Cantor-like set in Theorem 3.2 and Theorem 3.4. become the same value.

(2) Proposition 3.9 guarantees the positive Hausdorff dimension and correlation dimension of the set $F$.

The following example explains us that the regular condition in our symbolic construction need not satisfy the condition in Proposition 3.9.

Example 3.10. Consider a symbolic set $\mathcal{S}=\{1,2\}^{\mathbb{N}}$ satisfying conditions in section 2. Take a sequence $\left\{r_{\mathbf{i}_{n}}: \mathbf{i}_{n} \in\{1,2\}^{n}, n \geq 1\right\}$ such that $r_{\mathbf{i}_{n}}=r_{n}=\left(\frac{1}{4}\right)^{n}+\delta(0<\delta<1)$ for all $n=1,2,3, \ldots$ For all 
$\mathbf{i}=\left(i_{1}, i_{2}, \ldots\right) \in \mathcal{S}$ and all $n, j \geq 1$, we can not find the constant $M$ satisfying the condition in Proposition 3.9. On the other hand, for fixed $r>0$ and $x=\Pi(\mathbf{i})$ for $\mathbf{i}=\left(i_{1}, i_{2}, i_{3}, \ldots\right) \in \mathcal{S}$, there exists an integer number $n_{0} \equiv n_{0}(\mathbf{i})$ such that $\mathbf{i} \in C_{i_{1} \ldots i_{n_{0}+1}}$ and $r_{i_{1}} \cdots r_{i_{n_{0}+1}} \leq r<r_{i_{1}} \cdots r_{i_{n_{0}}}$. So the open ball $B(x, r)$ intersects at most two images for the map $\Pi$ of such cylinders at $n_{0}$-th step. Then the number $N(x, r)$ is less than or equal to 2 independent of $x$ and $r$.

\section{ACKNOWLEDGEMENT}

This research was supported by Basic Science Research Program through the National Research Foundation of Korea(NRF) funded by the Ministry of Education, Science and Technology(2012-0004725).

\section{References}

[1] I. S. Baek, Dimensions of weakly convergent deranged Cantor sets, Real Analysis Exchange, 23(2), (1997-98), 689-696.

[2] H. Baek, Packing dimension and measure of homogeneous Cantor sets, Bulletin of Aust. Math. Soc., 74(2006), 443-448.

[3] P. Billingsley, Ergodic Theory and Information, John Wiley \& Sons, (1965).

[4] C. A. Cabrelli, K. E. Hare and U. M. Molter, Sums of Cantor sets, Ergod. Th. \& Dynam. Sys. 17 (1997), 1299-1313.

[5] S. K. Chang, M. R. Lee and H. H. Lee, Bounds of correlation dimensions for snapshotattractors, Bull. Korean Math. Soc., 41(2) (2004), 327-335.

[6] K. Falconer, Techniques in fractal geometry, Mathematical Foundations and Applications, John Wiley \& Sons (1997).

[7] K. Falconer, Generalized dimensions of measures on almost self-affine sets, Nonlinearity, 23(5)(2010), 1047-1070.

[8] P. Grassberger and I. Procaccia, Characterrzation of Strange Attractors, Physical Review Letters, 50(3)(1983), 346-349.

[9] P. Grassberger, I. Procaccia, Measuring the strangeness of strange attractors, Physica D: Nonlinear Phenomena, 9(1-2)(1983), 189-208.

[10] K. E. Hare and S. Yazdani, Quasi self-similarity and multi-fractal analysis of Cantor measures, Real Analysis Exchange, 27(1), (2001/2002), 287-308.

[11] H.G.E. Hentschel, I. Procaccia, The infinite number of generalized dimensions of fractals and strange attractors, Physica D: Nonlinear Phenomena, 8(3)(1983), 435-444.

[12] J. E. Hutchinson, Fractals and self-similarity, Indiana Math. J., 30(1981), 713747.

[13] M. R. Lee and S. K. Chang, Dimensions for random loosely self-similar sets, Korean J. Math. Sciences, 9(2002), 1-8.

[14] M. R. Lee and H. H. Lee, Correlation dimensions of Cantor-like sets, Commun. Korean Math. Soc., 18(2)(2003), 281-288. 
[15] Curt McMullen, The Hausdorff dimension of general Sierpinski carpets, Nagoya Math. J., 96(1984), 1-9.

[16] J. Myjak, T. Szarek, On the Hausdorff dimension of Cantor-like sets with overlaps, Chaos, Solitons \& Fractals, 18(2)(2003), 329-333.

[17] E. Ott Chaos in Dynamical Systems, Cambridge Univercity Press, (1993)

[18] Y. Pesin and H. Weiss, On the dimension of deterministic and random Cantor-like sets, symbolic dynamics, and the Eckmann-Ruelle conjecture, Commun. Math. Phys., 182, (1996), 105-153.

[19] C. Q. Qu, H. Rao. and W. Y. Su, Hausdorff measure of homogenous Cantor set, Acta Math. Sinica, English Series, 17(1)(2001), 15-20.

[20] T. D. Sauer and J. A. Yorke, Are the dimensions of a set and its images equal under typical smooth functions ?, Ergod. Th. \& Dynam. Sys., 17(1997), 941-956.

[21] K. Simon and B. Solomyak, Correlation dimension for self-similar Cantor sets with overlaps, Fund. Math., 155(3)(1998), 293-300.

Mi Ryeong Lee

Department of Mathematics, Kyungpook National University,

Daegu 702-701, Korea.

E-mail: leemr@knu.ac.kr

Hunki Baek

Department of Mathematics Education, Catholic University of Daegu, Gyeongsan 712-702, Korea.

E-mail: hkbaek@cu.ac.kr 